\title{
Utilidad de la anamnesis y de las técnicas inmunológicas en el diagnóstico de la anisakidosis en pacientes con abdomen agudo
}

\author{
A. del Rey-Moreno, A. Valero-López¹, B. Gómez-Pozo², C. Mayorga-Mayorga ${ }^{3}$, J. Hernández-Quero ${ }^{4}$, \\ M. L. Garrido-Torres-Puchol ${ }^{5}$, M. J. Torres-Jaén ${ }^{3}$ y J. Lozano-Maldonado ${ }^{1}$

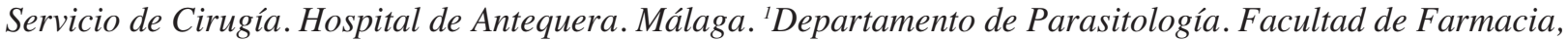 \\ Universidad de Granada. ${ }^{2}$ Universidad de Newcastle upon Tyne. ${ }^{3}$ Unidad de Investigación. Hospital Carlos Haya. \\ Málaga. ${ }^{4}$ Unidad de Enfermedades Infecciosas. Facultad de Medicina. Universidad de Granada. ${ }^{5}$ Unidad de \\ Inmunología. Hospital de Antequera. Málaga
}

\section{RESUMEN}

Introducción: Anisakis simplex puede producir síntomas digestivos y alérgicos. En este trabajo se evalúan los antecedentes epidemiológicos y los resultados inmunológicos para diferenciar entre pacientes con anisakidosis y aquellos con otras patologías digestivas que cursan con dolor abdominal.

Pacientes y método: estudio de cohortes realizado con 134 pacientes: 52 fueron diagnosticados de anisakidosis por los hallazgos quirúrgicos y anatomopatológicos y/o seroconversión específica frente a A. simplex (grupo A) y en 82 pacientes la anisakidosis fue excluida como diagnóstico (grupo NA: no-anisakidosis). Se han evaluado el antecedente de ingesta de pescado crudo, la prueba cutánea en prick (PC) y el inmunoblot IgE como elementos de diagnóstico.

Resultados: los pacientes de los grupos A y NA mostraron resultados diferentes respecto a la de ingesta de pescado crudo $(\mathrm{p}<$ $0,0001)$ y la PC ( $<<0,0001)$, con valores predictivos negativos (VPN) del 98,39 y $95,56 \%$ y positivos (VPP) del 70,83 y 87,50\%, respectivamente. En el inmunoblot, se halló una banda de aproximadamente $60 \mathrm{kDa}$ en el 86,2 y 19,2\% de los pacientes del grupo A y NA, respectivamente (VPP: 62,50\%; VPN: 94,03\%).

Conclusiones: en pacientes con dolor abdominal, la ingesta de pescado crudo o poco cocinado tiene elevada sensibilidad y VPN (98,39\%) pero menor VPP $(70,83 \%)$, por lo que nos ayuda principalmente a descartar la anisakidosis. La ausencia de sensibilización cutánea al extracto crudo de A. simplex apoya la ausencia de anisakidosis con una probabilidad alta $(95,56 \%)$. La presencia de una banda de $60 \mathrm{kDa}$ en el inmunoblot podría ser útil para su diagnóstico.

\begin{abstract}
Introduction: Anisakis simplex can be a cause of digestive symptoms. Our aim was to evaluate the epidemiological antecedents and immunological data available for a differentiation between patients with anisakidosis and those with other acute abdominal problems.

Patients and methods: this is a prospective cohort study involving 134 patients with acute abdominal problems: 52 patients were diagnosed with anisakidosis by means of surgical and pathological findings and/or specific IgE seroconversion against Anisakis simplex (group A), and in 82 patients anisakidosis had been ruled out (group NA: non-anisakidosis). We evaluated the antecedent of raw fish ingestion, the skin prick test, and $\operatorname{IgE} \mathrm{im}-$ munoblotting as diagnostic tools.

Results: patients in groups A and NA differ in terms of prior raw fish ingestion $(p<0.0001)$ and positive SPT $(p<0.0001)$, with their respective negative predictive values (NPV) being 98.39\% (95\%CI: 90.17-99.92) and 95.56\% (95\%CI: $83.64-$ 99.23). Regarding immunoblotting, in $86.2 \%$ of patients in group $\mathrm{A}$ a band of $60 \mathrm{kDa}$ was detected, which was also detected in $19.2 \%$ of patients in group NA.

Conclusions: a negative answer to the question about raw or undercooked fish ingestion has very high sensitivity and NPV (98.39\%), and is thus reasonably reliable to rule out anisakidosis. The absence of cutaneous sensitization to crude A. simplex extract gives a high probability (95.56\%) that the illness is absent. The presence of a band of about $60 \mathrm{kDa}$ in immunoblotting would be useful for diagnosis.
\end{abstract}

Key words: Anisakidosis. Anisakis simplex. Acute abdominal pain. Anamnesis. Skin prick test. Specific IgE. Immunoblotting.
Palabras clave: Anisakidosis. Anisakis simplex. Abdomen agudo. Anamnesis. Prueba cutánea. IgE específica. Inmunoblot.

Del Rey-Moreno A, Valero-López A, Gómez-Pozo B, Mayorga-Mayorga C, Hernández-Quero J, Garrido-Torres-Puchol ML, TorresJaén MJ, Lozano-Maldonado J. Utilidad de la anamnesis y de las técnicas inmunológicas en el diagnóstico de la anisakidosis en pacientes con abdomen agudo. Rev Esp Enferm Dig 2008; 100: 146-152.

Recibido: 05-09-07.

Aceptado: 08-01-08.

Correspondencia: Arturo del Rey-Moreno. Servicio de Cirugía General. Hospital de Antequera. C/ Poeta Muñoz Rojas, s/n. 29200 Antequera, Málaga. e-mail: arturodelreymoreno@gmail.com 


\section{INTRODUCCIÓN}

El complejo Anisakis simplex, la principal especie causante de la anisakidosis humana, tiene una distribución extensa. Se transmite al ser humano mediante la ingestión de pescado parasitado crudo o poco cocinado. El hombre se ha considerado un huésped paraténico, sin embargo, este concepto puede cambiar porque no sólo larvas en estadio III de A. simplex, sino también en proceso de muda a estadio IV han sido causantes de esta enfermedad $(1,2)$. Este parásito tiene varias formas de presentación: gástrica, intestinal, extra-gastrointestinal (heteróloga o ectópica), gastroalérgica y reacciones de hipersensibilidad mediadas por IgE, esta última también producida tras la ingestión de pescado adecuadamente cocinado (3-5), hecho puesto en duda por algunos autores (6). El curso clínico de la anisakidosis digestiva puede ser agudo y autolimitado, subagudo o crónico y, probablemente en la mayoría de los casos, oligosintomático $(7,8)$.

El diagnóstico de esta parasitosis es difícil debido a la ausencia de síntomas y signos clínicos característicos $(9,10) \mathrm{y}$, por ello, depende principalmente de la anamnesis, de la sospecha clínica y del seguimiento de los pacientes (11). El diagnóstico se confirma al observar la larva, cuando es expectorada o extraída mediante endoscopia de zonas accesibles (esófago, estómago, colon); sin embargo, es más difícil cuando la larva migra hacia el intestino delgado o es extra-gastrointestinal, requiriendo con frecuencia cirugía $(2,12-17)$, o cuando la infestación se hace crónica y la larva es destruida por el sistema inmune (7,18-20). El diagnóstico inmunológico se basa en la prueba cutánea con extracto crudo de larva de A. simplex y en la determinación de IgE específica frente a este (2124). La utilización de alérgenos purificados de excreciónsecreción (Ani s 1, Ani s 4) $(25,26)$, anticuerpos monoclonales (UA3, que reconoce el alérgeno Ani s 7) (27) y la medición de proteína catiónica del eosinófilo (28) han mejorado el diagnóstico de esta parasitosis, principalmente en las formas alérgicas.

La forma clínica intestinal se caracteriza por dolor abdominal agudo con una evolución generalmente favorable. En la práctica se puede resolver de dos maneras: a) tratamiento conservador en pacientes con dolor abdominal intenso y resultados patológicos en las exploraciones complementarias; o b) cirugía. Por ello, este estudio intenta evaluar la anamnesis y los medios de diagnóstico inmunológico disponibles para diferenciar entre pacientes con anisakidosis y aquellos con otras causas de abdomen agudo.

\section{PACIENTES Y MÉTODOS}

\section{Pacientes}

Este es un estudio de cohortes en pacientes atendidos en el Servicio de Urgencias del Hospital de Antequera (Málaga, España) en un periodo de 4 años y que requirie- ron ingreso por abdomen agudo, algunos de los cuales precisaron cirugía.

Los criterios de inclusión se detallan en la tabla I. Los sujetos se dividieron en 2 grupos tras un periodo de seguimiento: grupo A [52 pacientes: anisakidosis (resumen de datos en la tabla II)] y grupo NA (82 pacientes en los que la anisakidosis fue descartada: no anisakidosis). Todos los pacientes han formado parte del estudio tras ser informados y obtenido su consentimiento por escrito.

Tabla I. Criterios de inclusión de los grupos anisakidosis y no-anisakidosis

\begin{tabular}{ll}
\hline Grupo anisakidosis & Grupo no-anisakidosis \\
\hline -Dolor abdominal & -Dolor abdominal \\
-Hallazgos quirúrgicos y/o anatomo- & -Hallazgos radiológicos, quirúrgicos o ana- \\
patológicos de enteritis eosinofilica con & tomopatológicos relacionados con procesos \\
o sin la presencia de larva de anisákido, y/o & diferentes a la enteritis eosinofílica, y/o \\
-Seroconversión específica: cambio de clase & -Ausencia de seroconversión en el título de \\
en los valores de lgE específica frente a & IgE específica frente a A. simplex \\
A. simplex en determinaciones seriadas hasta & \\
6 meses después de la fase aguda de la & \\
enfermedad & \\
\hline
\end{tabular}

\section{Metodología}

Todos los individuos fueron evaluados en el Servicio de Urgencias del Hospital de Antequera. Se evaluaron los siguientes apartados:

- Anamnesis. Se preguntó a cada paciente por el antecedente de ingesta de pescado crudo o poco cocinado antes del comienzo de los síntomas digestivos.

- Prueba cutánea. Realizada con antígenos somáticos de A. simplex con una concentración de $2 \mathrm{mg} / \mathrm{ml}$ (ALKAbelló $^{\circledast}$, Madrid, España). Se consideró positiva cuando el diámetro de la pápula fue superior a $3 \mathrm{~mm}$. Como control positivo se utilizó histamina $(10 \mathrm{mg} / \mathrm{ml})$ y como control negativo $\mathrm{NaCl}$ al $0,9 \%$. Esta prueba sólo se pudo realizar en los dos últimos años.

-Determinación de IgE específica frente a A. simplex. Realizado mediante radioinmunoensayo (CAP system ${ }^{\circledast}$. Unicap Specific IgE, Pharmacia, Uppsala, Suecia). Fue determinada en la consulta de urgencias y entre 1-6 meses después de la fase aguda (la mayoría en los 2 primeros meses). Se consideraron positivos los resultados mayores de $0,35 \mathrm{kU} / 1$, según las normas del fabricante: clase $0<0,35$; clase 1: $0,35-<0,7$; clase $2: 0,7-<3,5$; clase $3: 3,5-<17,7$; clase 4: 17,7-<50; clase 5: 50-<100; clase 6: $\geq 100$.

-Detección de IgE específica mediante inmunoblot. La electroforesis proteica se realizó en gel de poliacrilamida (método de Laemmli) (29). El antígeno de extracto crudo de larva de A. simplex (ALK-Abelló ${ }^{\circledast}$, Madrid, España) se mezcló en una proporción $3: 1$ calentado a $100{ }^{\circ} \mathrm{C}$ durante $5 \mathrm{mi}-$ nutos y sometido a electroforesis en gel SDS-poliacrilamida al $12 \%$. Tras el proceso SDS-PAGE del extracto crudo de la larva, las proteínas separadas fueron transferidas en una 
membrana de nitrocelulosa (Pharmacia, Uppsala, Suecia) en un sistema Mini-Protean II (Bio-Rad ${ }^{\circledR}$, Richmond, California, EE.UU.). Tras bloqueo, con polvo de leche sin grasa al $5 \% \mathrm{w} / \mathrm{v}$, las membranas se incubaron durante la noche con $600 \mu \mathrm{l}$ de suero de los pacientes diluidos al 1:5. Después de tres lavados, las membranas se incubaron con anticuerpo monoclonal anti-IgE marcado con peroxidasa (Sigma, St. Louis, Missouri, EE.UU.) diluido al 1:600. Para finalizar, la detección fue realizada mediante método de quimioluminescencia (ECL ${ }^{\oplus}$, Amershan Pharmacia Biotech).

\section{Análisis estadístico}

Se ha realizado con EPIINFO 3.3.2 y Epidat 3.1. En las variables cuantitativas con distribución normal se utilizó la prueba t de Student y la prueba U de Mann-Whitney en las de distribución no normal. En las variables categóricas se han utilizado la Chi cuadrado de Pearson y el índice kappa.

\section{RESULTADOS}

En este estudio se han incluido 134 pacientes. En el grupo A, 23 de los 52 pacientes fueron operados -4 de ellos perforados: 1 de localización gástrica, 2 ileal y 1 de colon derecho-, los 29 restantes fueron diagnosticados tras observar seroconversión de la IgE específica (Tabla II). El grupo NA está formado por 82 pacientes con abdomen agudo de diferente etiología: apendicitis aguda, colecistitis aguda, otras causas de oclusión intestinal, etc.

Tabla II. Resumen de datos de los pacientes del grupo anisakidosis

\begin{tabular}{|c|c|c|c|c|c|c|c|}
\hline Paciente & Tratamiento & $\lg E A S 1$ & $\operatorname{lgE} A S 2$ & Localización & $P C(\mathrm{~mm})$ & Parásito & Diagnóstico preoperatorio \\
\hline $\begin{array}{l}1 \\
2 \\
3 \\
4 \\
5 \\
6 \\
7 \\
8 \\
9 \\
10 \\
11 \\
12 \\
13 \\
14 \\
15 \\
16 \\
17 \\
18 \\
19 \\
20 \\
21 \\
22 \\
23 \\
24 \\
25 \\
26 \\
27 \\
28 \\
29 \\
30 \\
31 \\
32 \\
33 \\
34 \\
35 \\
36 \\
37 \\
38 \\
39 \\
40 \\
41 \\
42 \\
43 \\
44 \\
45 \\
46 \\
47 \\
48 \\
49 \\
50 \\
51 \\
52\end{array}$ & $\begin{array}{l}\text { Quirúrgico } \\
\text { Quirúrgico } \\
\text { Quirúrgico } \\
\text { Quirúrgico } \\
\text { Quirúrgico } \\
\text { Quirúrgico } \\
\text { Quirúrgico } \\
\text { Quirúrgico } \\
\text { Quirúrgico } \\
\text { Quirúrgico } \\
\text { Quirúrgico } \\
\text { Quirúrgico } \\
\text { Quirúrgico } \\
\text { Quirúrgico } \\
\text { Conservador } \\
\text { Conservador } \\
\text { Quirúrgico } \\
\text { Conservador } \\
\text { Conservador } \\
\text { Quirúrgico } \\
\text { Conservador } \\
\text { Conservador } \\
\text { Quirúrgico } \\
\text { Conservador } \\
\text { Quirúrgico } \\
\text { Conservador } \\
\text { Quirúrgico } \\
\text { Conservador } \\
\text { Conservador } \\
\text { Conservador } \\
\text { Conservador } \\
\text { Quirúrgico } \\
\text { Quirúrgico } \\
\text { Conservador } \\
\text { Conservador } \\
\text { Conservador } \\
\text { Conservador } \\
\text { Quirúrgico } \\
\text { Quirúrgico } \\
\text { Conservador } \\
\text { Conservador } \\
\text { Conservador } \\
\text { Conservador } \\
\text { Conservador } \\
\text { Conservador } \\
\text { Conservador } \\
\text { Conservador } \\
\text { Conservador } \\
\text { Conservador } \\
\text { Conservador } \\
\text { Conservador } \\
\text { Conservador }\end{array}$ & $\begin{array}{c}73,8 \\
100 \\
5,36 \\
14,2 \\
30,5 \\
96 \\
16,6 \\
50,3 \\
75,1 \\
52 \\
8,27 \\
86,6 \\
100 \\
2,38 \\
21,6 \\
32,3 \\
15,6 \\
72,6 \\
13,4 \\
3,24 \\
3,88 \\
25,9 \\
1,78 \\
12,8 \\
2,36 \\
4,43 \\
4,22 \\
13,5 \\
18,7 \\
14 \\
16,6 \\
100 \\
0,47 \\
32,8 \\
12,6 \\
2,43 \\
51,8 \\
1,63 \\
0 \\
20 \\
20,4 \\
19,6 \\
51,5 \\
2 \\
71,2 \\
20,3 \\
1,14 \\
18,3 \\
34,1 \\
12,8 \\
1,48 \\
2,03\end{array}$ & $\begin{array}{l}10,4 \\
60,4 \\
3,97 \\
3,72 \\
77,2 \\
82,9 \\
1,26 \\
9,12 \\
31,5 \\
31,83 \\
46,1 \\
96,2 \\
1,37 \\
3,23 \\
0,72 \\
9,44 \\
5,48 \\
6,11 \\
61,8 \\
5,74 \\
2,57 \\
21,6 \\
9,98 \\
2,7 \\
2,43 \\
3,2 \\
9,4 \\
22 \\
0 \\
40,3 \\
23,8 \\
9,7 \\
70,6 \\
23,9 \\
42,1 \\
12,9 \\
4,82 \\
0,83 \\
8,83 \\
13,5 \\
98,6 \\
10 \\
10,5 \\
4,84 \\
10,6 \\
62,6 \\
75,7 \\
71,7 \\
31,8 \\
45,4 \\
\end{array}$ & $\begin{array}{c}\text { Yeyuno } \\
\text { Yeyuno } \\
\text { Yeyuno } \\
\text { Colon derecho y transverso } \\
\text { colon derecho } \\
\text { Colon derecho } \\
\text { lleon } \\
\text { lleon } \\
\text { Yeyuno } \\
\text { lleon } \\
\text { Íleon } \\
\text { Colon derecho } \\
\text { Îleon } \\
\text { íleon } \\
\\
\text { Íleon } \\
\text { Yeyuno } \\
\text { Íleon } \\
\text { Colon derecho } \\
\text { Íleon }\end{array}$ & $\begin{array}{l}2 \\
\\
4 \\
5 \\
0 \\
7 \\
4 \\
5 \\
\\
5 \\
8\end{array}$ & $\begin{array}{l}\text { Sí } \\
\text { Sí }\end{array}$ & $\begin{array}{l}\text { Obstrucción intestinal } \\
\text { Obstrucción intestinal } \\
\text { Abdomen agudo } \\
\text { Plastrón inflamatorio } \\
\text { Apendicitis aguda } \\
\text { Obstrucción intestinal } \\
\text { Obstrucción intestinal } \\
\text { Abdomen agudo } \\
\text { Abdomen agudo } \\
\text { Obstrucción intestinal } \\
\text { Obstrucción intestinal } \\
\text { Apendicitis aguda } \\
\text { Obstrucción intestinal } \\
\text { Plastrón inflamatorio } \\
\text { Abdomen agudo } \\
\text { Apendicitis aguda } \\
\text { Obstrucción intestinal } \\
\text { Apendicitis aguda } \\
\text { Apendicitis aguda }\end{array}$ \\
\hline
\end{tabular}
parásito en pieza quirúrgica. 
La edad media de los pacientes del grupo A [42,9 \pm $12,7(\chi \pm \mathrm{DE})]$ y del grupo NA $(47,1 \pm 20,3)$ no presentó diferencias significativas $(\mathrm{p}=0,2811)$. Respecto a la distribución por sexos tampoco hubo diferencias significativas $(\mathrm{p}=0,354)$.

Todos excepto uno de los pacientes del grupo A $(98,1 \%)$ confirmaron la ingesta de pescado crudo o poco cocinado: boquerones frescos, crudos o en vinagre (50 pacientes) y sardinas crudas pequeñas (1 paciente); ningún otro tipo de pescado ha sido causante de la enfermedad en este estudio. En el grupo NA, 21 pacientes $(25,6 \%)$ ingirieron pescado crudo o poco cocinado antes del comienzo del dolor abdominal (Tabla III), mostrando este antecedente epidemiológico diferencia significativa entre ambos grupos ( $<<0,0001)$, con un VPP del 70,83\% (IC95\%: 58,77-80,65) y un VPN de 98,39\% (IC95\%: 90,17-99,92) (Tablas III y VI).

Tabla III. Resultados de la anamnesis

\begin{tabular}{lccc}
\hline Ingestión de pescado crudo & Grupo A (\%) & Grupo NA (\%) & Total \\
\hline Sí & $51(98,1 \%)$ & $21(25,6 \%)$ & 72 \\
No & $1(1,9 \%)$ & $61(74,4 \%)$ & 62 \\
\hline
\end{tabular}

Hemos realizado gastroscopia a 4 pacientes del grupo A, todos con hallazgos patológicos (2 con úlcera gástrica y 2 con gastritis), 3 de ellos con dilatación de asas intestinal y/o presencia de líquido libre intraabdominal -es decir, de localización probablemente intestinal- y 1 con neumoperitoneo radiológico, motivo por el que hubo de ser intervenido.

La prueba cutánea no estuvo disponible hasta el año 2000 , por ello sólo se realizó en la fase aguda de la enfermedad a 16 pacientes $(30,76 \%)$ del grupo A y a 45 $(54,87 \%)$ del grupo NA. Respecto a esta variable, se halló diferencia significativa entre ambos grupos $(\mathrm{p}<0,0001)$ (Tabla IV), con VPP del 87,50\% (IC95\%: 60,41-97,80) y VPN que alcanzó el 95,56\% (IC95\%: 83,64-99,23) (Tabla V). En el grupo NA, la PC fue positiva en 2 pacientes, uno con apendicitis aguda y el otro con diverticulitis aguda que habían ingerido boquerones en vinagre 48 horas antes del comienzo de los síntomas digestivos. En los pacientes del grupo NA que no habían ingerido pescado crudo, la PC resultó en todos negativa ( 0 de 28 pacientes). En los pacientes de ambos grupos que habían ingerido pescado crudo, el VPP de este test ha sido de 87,50\% (IC95\%: 60,41-97,80) y el VPN de 88,24\% (IC95\%: 62,25-97,94).

Tabla IV. Resultados de las pruebas cutáneas

\begin{tabular}{lccc}
\hline Prueba cutánea & Grupo A (\%) & Grupo NA (\%) & Total \\
\hline Positiva & $14(87,5 \%)$ & $2(4,4 \%)$ & 16 \\
Negativa & $2(12,5 \%)$ & $43(95,6 \%)$ & 45 \\
\hline
\end{tabular}

En el inmunoblot realizado con suero de los pacientes en la fase aguda de la enfermedad se observaron bandas antigénicas con pesos moleculares desde < 30 a 205 kDa. En los pacientes con anisakidosis la mayoría de bandas antigénicas se encontraban entre los pesos moleculares 44 y 82 kDa, y en el grupo NA, entre 82 y 119 kDa. Una banda intensa de aproximadamente $60 \mathrm{kDa}$ fue detectada en el inmunoblot del $86,2 \%$ de los pacientes en el grupo A, que también se observó en el 19,2\% de los pacientes del grupo NA (Tabla V) (Fig. 1). En los pacientes en los que se hallaron parásitos durante la cirugía -5 pacientes del grupo A-, el perfil de bandas del inmunoblot fue similar al del resto de pacientes del grupo A. Uno de estos, de localización yeyunal, en el que se determinó la IgE específica y se realizó inmunoblot inmediatamente después de la cirugía y, posteriormente, al alta hospitalaria $\left(5^{\circ}\right.$ día de postoperatorio), se observó una elevación de la IgE específica de 0,4 a 79,7 y, en el inmunoblot, la banda de $60 \mathrm{kDa}$ que no fue visible inmediatamente tras la cirugía (i de figura 1), apareció previo al alta del paciente (j de figura 1). La presencia de la banda de $60 \mathrm{kDa}$ está relacionada con la elevación de IgE específica contra A. simplex $(0,648$; IC95\%: 0,497-0,799) y con la prueba cutánea positiva $(0,702$; IC95\%: 0,499-0,906). Las áreas bajo la curva ROC para los tres tests -anamnesis, PC e inmunoblot, se muestran en la figura 2 .

Tabla V. Resultados del inmunoblot

\begin{tabular}{lrcc}
\hline Inmunoblot & Grupo A (\%) & Grupoo NA (\%) & Total \\
\hline Presencia de banda de 60 kDa $25(86,2 \%)$ & $15(19,2 \%)$ & 40 \\
Ausencia de banda de 60 kDa & $4(13,8 \%)$ & $63(80,8 \%)$ & 67 \\
\hline
\end{tabular}

\section{DISCUSIÓN}

La anisakidosis debe ser sospechada para poder realizar su diagnóstico $(14,15,30)$ y, con toda probabilidad, está infradiagnosticada, pudiendo ser esta la causa de pacientes con síntomas dispépticos $(31,32)$ o dolor abdominal leve $(10,33)$. En nuestro estudio, una simple pregunta sobre la ingesta de pescado crudo ha sido muy útil, mostrando una elevada sensibilidad $(98,08 \%)$ y VPN $(98,39 \%)$, aunque su VPP ha sido menor $(70,83 \%)$ probablemente debido al elevado consumo de pescado crudo en la población (34). Es decir, si existe dicho antecedente nos puede orientar al diagnóstico pero es su ausencia la que descarta con probabilidad elevada esta patología. Un problema añadido es que el paciente puede no recordar la ingesta de pescado crudo $(4,9,30)$ debido al tiempo prolongado entre la ingestión de la larva y el comienzo de la sintomatología digestiva o alérgica, aunque en nuestro caso sólo hubo un falso negativo (1,9\%). Asaishi y cols. (9), en un estudio sobre pacientes con anisakidosis intestinal confirmada, observaron que sólo el $28 \%$ de los mismos relacionaban los síntomas con la ingesta de pescado crudo. 
En Japón, la mayoría de los casos de anisakidosis se deben a la ingesta de caballa cruda $(35,36)$, mientras que en el oeste de Europa es por ingesta de marinados de arenque $(11,37)$. En España, el principal pescado responsable son los boquerones frescos en vinagre, aunque también se han implicado otras especies: merluza, sardinas, bacalao, etc. $(4,6,27,32,38-40)$.

La mayoría de los enfermos vistos en nuestro servicio son pacientes con patología intestinal, ya que los pacientes con síntomas dispépticos o con dolor epigástrico son valorados en servicios médicos y sometidos a tratamiento médico (16).

La sensibilidad de la PC ha sido del 87,5\% (IC95\%: 60,4-97,8) y la especificidad del 95,6\% (IC95\%: 83,6$99,2)$ (Tabla VI), es decir, una PC negativa en un paciente con dolor abdominal, haya consumido o no pescado crudo, excluye con una probabilidad elevada $(95,56 \%)$ la presencia de anisakidosis y sugiere otras causas de dolor abdominal; aunque debemos tener en cuenta que su resultado puede ser negativo - al igual que la cifra de $\operatorname{IgE}$ específica o el inmunoblot- en el comienzo del cuadro clínico en pacientes que sufren una primoinfestación (2), probablemente debido a que no se ha desarrollado respuesta inmune en la fase precoz de la enfermedad, o puede ser falso positivo -ninguno en este estudio- por seroprevalencia elevada en la población sana, que se ha relacionado con la ingesta de pescado crudo, en vinagre o ahumado y la elevada frecuencia de consumo de pescado $(34,41)$.

La IgE específica contra $A$. simplex sólo es útil como orientación y, en caso de que un paciente presente dolor abdominal agudo, el dato único de elevación de esta no debe alterar el tratamiento planeado $(2,16)$.

En este estudio, la banda antigénica más útil en el inmunoblot para diagnóstico de anisakidosis es la que tiene un peso molecular aproximado de $60 \mathrm{kDa}$ (Fig. 1), observada en el 86,2\% de los pacientes del grupo A y en el $19,2 \%$ del grupo NA; su peso molecular es similar al de la banda hallada por Akao y cols. (23) en pacientes con anisakidosis gástrica. Es preciso enfatizar el papel de la anamnesis como medio diagnóstico para esta enfermedad, mejorando los resultados de exploraciones complementarias, como el inmunoblot (Tabla III) (Fig. 2); esta

Tabla VI. Capacidad predictiva de la anamnesis, prueba cutánea e inmunoblot

\begin{tabular}{lcrr}
\hline & Anamnesis (IC95\%) & Prueba cutánea (IC95\%) & Inmunoblot (IC95\%) \\
\hline Prevalencia de la enfermedad & $38,81 \%(30,63-47,64)$ & $26,23 \%(16,17-39,31)$ & $27,10 \%(19,18-36,70)$ \\
Seguridad diagnóstica & $83,58 \%(75,98-89,21)$ & $93,44 \%(83,25-97,88)$ & $82,24 \%(73,40-88,71)$ \\
Sensibilidad & $98,08 \%(88,42-99,90)$ & $87,50 \%(60,41-97,80)$ & $86,21 \%(67,43-95,49)$ \\
Especificidad & $74,39 \%(63,35-83,11)$ & $95,56 \%(83,64-99,23)$ & $80,77 \%(69,95-88,49)$ \\
VPP & $70,83 \%(58,77-80,65)$ & $87,50 \%(60,41-97,80)$ & $62,50 \%(45,81-76,83)$ \\
VPN & $98,39 \%(90,17-99,92)$ & $95,56 \%(83,64-99,23)$ & $94,03 \%(84,65-98,07)$ \\
CP+ & $3,83(2,64-5,55)$ & $19,69(5,02-77,27)$ & $4,48(2,78-7,23)$ \\
CP- & $0,03(0-0,18)$ & $0,13(0,04-0,48)$ & $0,17(0,07-0,43)$ \\
\hline
\end{tabular}

VPP: valor predictivo positivo; VPN: valor predictivo negativo; $\mathrm{CP}+$ : cociente de probabilidades positivo; $\mathrm{CP}$-: cociente de probabilidades negativo.

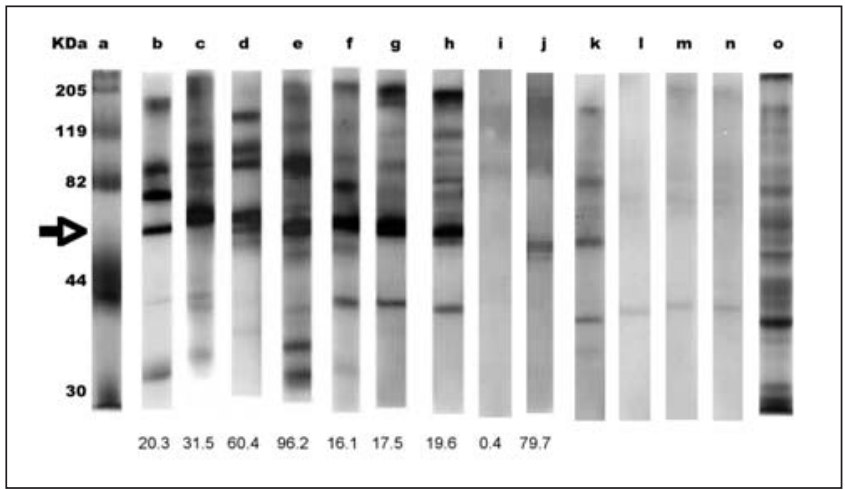

Fig. 1. Inmunoblot IgE con antígenos somáticos de $A$. simplex. a) marcador de peso molecular; $b$-j) sueros de pacientes del grupo A [con los valores de IgE específica (kUA/l) en su parte inferior]; $k$ ) suero de un paciente del grupo no-anisakidosis (3,27 kUA/L); I-n) sueros de pacientes del grupo no-anisakidosis en los que no se elevó la IgE específica frente a A. simplex; o: antígeno somático de $A$. simplex. La flecha indica la banda de aproximadamente $60 \mathrm{kDa}$.

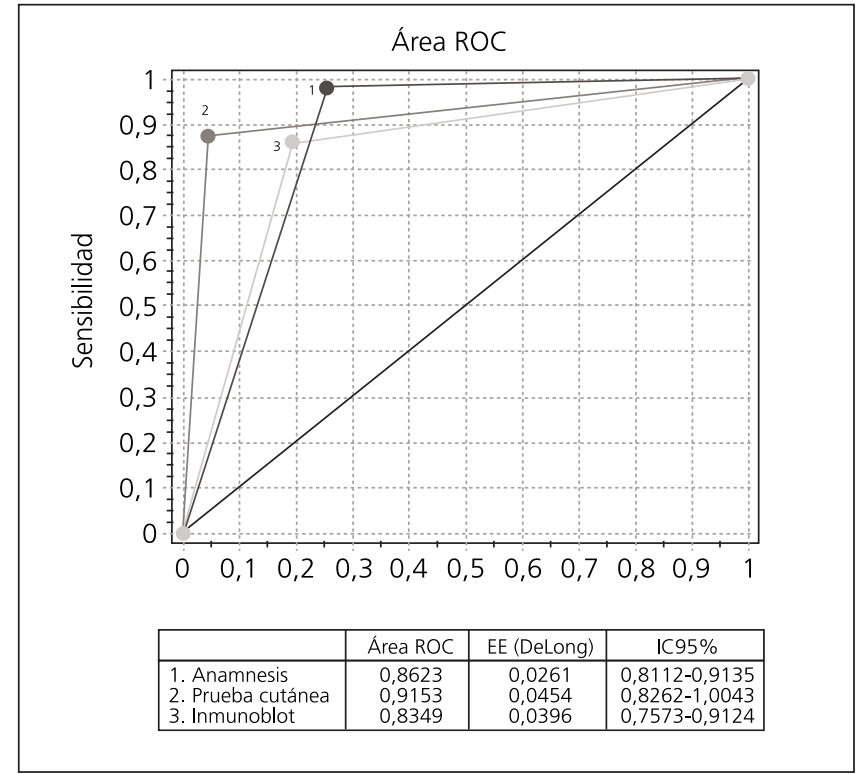

Fig. 2. Comparación de las áreas ROC entre anamnesis, prueba cutánea e inmunoblot. 
última prueba apoya los resultados de los otros dos -anamnesis y $\mathrm{PC}-$, si bien sus resultados se obtienen después de que el paciente ha sido dado de alta hospitalaria. Además, el patrón de reconocimiento de alérgenos es muy variable y depende de factores genéticos y del intervalo entre el contacto con el parásito y la extracción de muestra de sangre (42); por ello es posible que el perfil de anticuerpos detectados en un paciente con parasitismo reciente con reacción alérgica difiere del paciente sin historia de alergia o con síntomas subclínicos previos (30). En un trabajo realizado por Baeza y cols. (43) con pacientes alérgicos a $A$. simplex observaron que estos estaban sensibilizados a gran número de proteínas, tanto de excreción-secreción como somáticas, con gran variabilidad de reconocimiento de los alérgenos, sin existir ningún alérgeno reconocido por todos los pacientes.

Como observamos, el diagnóstico de esta parasitosis puede ser difícil, especialmente si el antecedente de ingesta de pescado crudo o poco cocinado no se tiene en cuenta. El resultado de la serología en pacientes con abdomen agudo no debe retardar ni cambiar la actitud terapéutica. En caso de que un paciente haya ingerido pescado crudo y presente dolor abdominal sin signos peritoneales deben realizarse exploraciones complementarias (laboratorio, imagen) y tener un seguimiento clínico en un medio quirúrgico. La serología se debe realizar, pero no debe retardar la actitud terapéutica por el riesgo: primero de que su etiología tenga un tratamiento quirúrgico y, segundo, de perforación u oclusión $(16,44,45)$, como en algunos de nuestros casos, a pesar de una evolución habitualmente favorable $(17,46)$.

El diagnóstico de esta enfermedad se puede mejorar mediante la determinación de la proteína catiónica de eosinófilo (28), la utilización de anticuerpos monoclonales (UA3) (27) o la realización de inmunoblot IgE e IgG frente a alergenos de excreción-secreción purificados (Ani s 1) $(25,47,48)$. Aunque los alérgenos secretados por el parásito son más potentes que los extractos somáticos (43), en un trabajo reciente de Moneo y cols. (49) con pacientes sensibilizados a A. simplex se ha observado que el extracto crudo fraccionado y concentrado o sin fraccionar obtuvo mejor sensibilidad e igual especificidad que las proteínas de excreción-secreción Ani s 1 y Ani s 4. Otra línea de investigación para el diagnóstico es la detección de antígenos circulantes de A. simplex, ya conseguido en anisakidosis experimental en ratas (50).

Finalmente, queremos remarcar respecto a los resultados de este trabajo, la importancia de la anamnesis y la correlación positiva entre la elevación de la IgE específica frente a $A$. simplex, la prueba cutánea positiva y la presencia de la banda de $60 \mathrm{kDa}$ en el inmunoblot.

\section{BIBLIOGRAFÍA}

1. Ishikura H, Takahashi S, Ishikura H. Anisakidae and anisakidosis in Japan. Proceed. $2^{\text {nd }}$ Japan-Korea Parasitologists. Seminar (Forum Cheju-2) 1996: 50-63.
2. Del Rey Moreno A. Estudio parasitológico, epidemiológico y clínico de la anisakidosis en la comarca de Antequera. Tesis Doctoral. Granada; 2003.

3. Audicana MT, Fernández de Corres L, Muñoz D, Fernández E, Navarro JA, del Pozo MD. Recurrent anaphylaxis caused by Anisakis simplex parasitizing fish. J Allergy Clin Immunol 1995; 96: 558-60.

4. Fernández de Corres L, Audícana M, del Pozo MD, Muñoz D, Fernández E, Navarro JA, et al. Anisakis simplex induces not only anisakiasis: Report on 28 cases of allergy caused by this nematode. J Invest Allergol Clin Immunol 1996; 6: 315-319.

5. Audicana MT, Ansotegui IJ, Fernández de Corres L, Kennedy MW Anisakis simplex: Dangerous-dead and alive? Trends Parasitol 2002; 18: $20-5$.

6. Alonso-Gómez A, Moreno-Ancillo A, López-Serrano MC, Suárezde-Parga JM, Daschner A, Caballero MT, et al. Anisakis simplex only provokes allergic symptoms when the whorm parasitises the gastrointestinal tract. Parasitol Res 2004; 93: 378-84.

7. Yokogawa M, Yoshimura H. Clinicopathologic studies on larval anisakiasis in Japan. Am J Trop Med Hyg 1967; 16: 723-8.

8. Daschner A, Alonso-Gómez A, Caballero T, Suárez de Parga JM, López-Serrano MC. Usefulness of early serial measurement of specific and total immunoglobulin $\mathrm{E}$ in the diagnosis of gastro-allergic anisakiasis. Clin Exp Allergy 1999; 29: 1260-4

9. Asaishi K, Nishino C, Totsuka M, Hayasaka H, Suzuki T. Studies on the etiologic mechanism of anisakiasis. 2. Epidemiologic study of inhabitants and questionaire survey in Japan. Gastroenterol Jpn 1980; 15: $128-34$

10. Sakanari JA, McKerrow JH. Anisakiasis. Clin Microbiol Rev 1989; 2: 278-84.

11. Verhamme MAM, Ramboer CHR. Anisakiasis caused by herring in vinegar: A little known medical problem. Gut 1988; 29: 843-7.

12. Smith JW, Wootten R. Anisakis and anisakiasis. Adv Parasitol 1978; 16: 93-163.

13. Valero A, Mira Gutiérrez J, Pérez Cano R, Sanz Domínguez J, Geraldía Lomas M, García Herruzo J, et al. Descripción de un caso de anisakiosis humana. IX Reunión Científica de Parasitólogos Españoles: Homenaje al Prof. Dr. Miguel Cordero del Campillo. León: 1992. p. 69.

14. Castán B, Borda F, Iñarrairaegui M, Pastor G, Vila J, Zozaya M. Anisakiasis digestiva: clínica y diagnóstico según localización. Rev Esp Enferm Dig 2002; 94: 463-67.

15. Repiso Ortega A, Alcántara Torres M, González de Frutos C, de Artaza Varasa T, Rodríguez Merlo R, Valle Muñoz J, et al. Anisakiasis gastrointestinal. Estudio de una serie de 25 pacientes. Gastroenterol Hepatol 2003; 26: 341-6.

16. Ortega-Deballón P, Carabias-Hernández A, Martín-Blázquez A, Garaulet $\mathrm{P}$, Benoit L, Kretz B, et al. Anisakiase: Une parasitose que le chirurgien doît connaître. Ann Chir 2005; 130: 407-10

17. Ramos L, Alonso C, Guilarte M, Vilaseca J, Santos J, Malagelada JR. Anisakis simplex-induced small bowel obstruction after fish ingestion: Preliminary evidence for response to parenteral corticosteroids. Clin Gastroenterol Hepatol 2005; 3: 667-71.

18. Petithory JC, Paugam B, Buyet-Rousset P, Paugam A. Anisakix simplex, a co-factor of gastric cancer? Lancet 1990; 336: 1002.

19. Kim H. Chronic gastric anisakiasis: Radiologic and endoscopic features. Am J Roentgenol 1994; 162: 468-9.

20. Ito Y, Ikematsu Y, Yuzawa H, Nishiwaki Y, Kida H, Waki S, et al Chronic gastric anisakiasis presenting as pneumoperitoneum. Asian J Surg 2007; 30: 67-71.

21. Del Pozo MD, Moneo I, Fernández de Corres L, Audicana MT, Muñoz D, Fernández E, et al. Laboratory determinations in Anisakis simplex allergy. J Allergy Clin Immunol 1996; 97: 977-84.

22. García M, Moneo I, Audicana MT, del Pozo MD, Muñoz D, Fernández E, et al. The use of IgE immunoblotting as a diagnostic tool in Anisakis simplex allergy. J Allergy Clin Immunol 1997; 99: 497-501.

23. Akao N, Ohyama $\mathrm{T}$, Kondo K. Immunoblot analysis of serum IgG, $\operatorname{IgA}$ and IgE responses against larval excretory-secretory antigens of Anisakis simplex in patients with gastric anisakiasis. J Helminthol 1990; 64: 310-8

24. Valero A, Terrados S, Díaz V, Reguera V, Lozano J. Determination of $\operatorname{IgE}$ in the serum of patients with allergic reactions to four species of fish-parasite anisakids. J Invest Allergol Clin Immunol 2003; 13: 94-8 
25. Moneo I, Caballero ML, Gómez F, Ortega E, Alonso MJ. Isolation and characterization of a major allergen from the fish parasite Anisakis simplex. J Allergy Clin Immunol 2000; 106: 177-82.

26. Rodríguez-Mahillo AI, González-Muñoz M, Gómez-Aguado F, Rodríguez-Pérez R, Corcuera MT, Caballero ML, et al. Cloning and characterisation of the Anisakis simplex allergen Ani s 4 as cysteine-protease inhibitor. Int J Parasitol 2007; 37: 900-17.

27. Iglesias R, Leiro J, Santamarina MT, Sanmartín ML, Ubeira FM. Monoclonal antibodies against diagnostic Anisakis simplex antigens. Parasitol Res 1997; 83: 755-61.

28. Domínguez-Ortega J, Martínez-Alonso JC, Alonso-Llamazares A, Argüelles-Grande C, Chamorro M, Robledo T, et al. Measurement of serum levels of eosinophil cationic protein in the diagnosis of acute gastrointestinal anisakiasis. Clin Microbiol Infect 2003; 9: 453-7.

29. Laemmli UK. Cleavage of estructural proteins during the assembly of the head on bacteriophage T4. Nature 1970; 227: 680-5.

30. Daschner A, Pascual CY. Anisakis simplex: Sensitization and clinical allergy. Curr Opin Allergy Clin Immunol 2005; 5: 281-5.

31. Toro C, Caballero MT, Baquero M, García-Samaniego J, Casado I, Rubio M, et al. High prevalence of seropositivity to a major allergen of Anisakis simplex, Ani s 1, in dyspeptic patients. Clin Diag Lab Immunol 2004; 11: 115-8.

32. Toro C, Caballero ML, Baquero M, García-Samaniego J, Casado I, Martínez P, et al. Seropositivity to a major allergen of Anisakis simplex, Ani s 1, in dyspeptic patients with Helicobacter pylori infection Histological and laboratory findings and clinical significance. Clin Microbiol Infect 2006; 12: 453-8.

33. Gutiérrez Ramos R, Tsuji M. Detection of antibodies to Anisakis simplex larvae by enzyme-linked immunosorbent assay and immunoelectrophoresis using crude or purified antigens. J Helminthol 1994; 68: 305-9.

34. Del Rey Moreno A, Valero A, Mayorga C, Gómez B, Torres MJ, Hernández J, et al. Sensitization to Anisakis simplex s.l. in a healthy population. Acta Tropica 2006; 97: 265-9.

35. Nakata H, Takeda K, Nakayama T. Radiological diagnosis of acute gastric ansakiasis. Radiol 1980; 135: 49-53.

36. Sugimachi K, Inokuchi K, Ooiwa T, Fujino T, Ishii Y. Acute gastric anisakiasis. Analysis of 178 cases. JAMA 1985; 253: 1012-3.

37. Mudry J, Lefebvre P, Dei-Cas E, Vernes A, Poirriez J, Débat M, et al Anisakiase humaine: 5 cas dans le nord de la France. Gastroenterol Clin Biol 1986; 10: 83-7.

38. Domínguez Ortega J, Cimarra M, Sevilla MC, Alonso Llamazares A, Moneo I, Robledo Echarren T, et al. Anisakis simplex: una causa de pseudobstrucción intestinal. Rev Esp Enferm Dig 2000; 92: 132-5.

39. López Peñas D, Ramírez Ortiz LM, del Rosal Palomeque R, López Rubio F, Fernández-Crehuet Navajas R, Miño Fugarolas G. Estudio de trece casos de anisakiasis en la provincia de Córdoba. Med Clin (Barc) 2000; 114: 177-80

40. González Quijada S, González Escudero R, Arias García L, Gil Martín AR, Vicente Serrano J, Corral Fernández E. Manifestaciones digestivas de la anisakiasis: descripción de 42 casos. Rev Clin Esp 2005; 7: 311-5.

41. Puente P, Anadón AM, Rodero M, Romarís F, Ubeira FM, Cuéllar C. Anisakis simplex: The high prevalence in Madrid (Spain) and its relation with fish consumption. Exp Parasitol 2007. Available at: doi:10.1016/j.expara.2007.07.002.

42. Daschner A, Cuéllar C, Sánchez-Pastor S, Pascual CY, Martín-Esteban M. Gastro-allergic anisakiasis as consequence of simultaneous primary and secondary immune response. Parasite Immunol 2002; 24: 243-51.

43. Baeza ML, Rodríguez A, Matheu V, Rubio M, Tornero P, de Barrio $\mathrm{M}$, et al. Characterization of allergens secreted by Anisakis simplex parasite: Clinical relevance in comparison with somatic allergens. Clin Exp Allergy 2004; 34: 296-302

44. Magarzo García J, Moral Duarte A, Serrano Escobedo A, Chierichetti Asan C, Artigas Raventós V, Trias Folch M. Perforación intestinal por anisakiasis. Cir Esp 2002; 72: 365-6.

45. Matsuo S, Azuma T, Susumu S, Yamaguchi S, Obata S, Hayasi T. Small bowel anisakidosis: A report of two cases. World J Gastroenterol 2006; 12: 4106-8.

46. Ponferrada A, Matilla A, Borrego GM, Becerro I, Núñez O, Lamónaca M, et al. Hemoperitoneo espontáneo secundario a yeyunoileítis por Anisakis. Rev Esp Enferm Dig 2005; 97: 292-3

47. Caballero ML, Moneo I. Specific IgE determination to Ani s 1, a major allergen from Anisakis simplex, is a useful tool for diagnosis. Ann Allergy Asthma Immunol 2002; 89: 74-7

48. Shimakura K, Miura H, Ikeda K, Ishizaki S, Nagashima Y, Shirai T, et al. Purification and molecular cloning of a major allergen from Anisakis simplex. Mol Biochem Parasitol 2004; 135: 69-75.

49. Moneo I, Caballero ML, Rodríguez-Pérez R, Rodríguez-Mahillo AI, González-Muñoz M. Sensitization to the fish parasite Anisakis simplex: Clinical and laboratory aspects. Parasitol Res 2007; 101: 1051-5.

50. Campos M, Martín L, Díaz V, Mañas I, Morales B, Lozano J. Detection of circulating antigens in experimental anisakiasis by two-site enzymelinked immnunosorbent assay. Parasitol Res 2004; 93: 433-8. 